\title{
Enhanced Recovery Pathways for Cardiac Surgery
}

\author{
Scott R. Coleman ${ }^{1} \cdot$ Ming Chen ${ }^{2} \cdot$ Srikant Patel ${ }^{1} \cdot$ Hong Yan $^{3}$ - Alan D. Kaye ${ }^{4}$ - Marcus Zebrower ${ }^{1} \cdot$ Julie A. Gayle ${ }^{4}$ \\ Henry Liu ${ }^{1} \cdot$ Richard D. Urman ${ }^{5}$
}

Published online: 14 March 2019

(C) Springer Science+Business Media, LLC, part of Springer Nature 2019

\begin{abstract}
Purpose of Review Enhanced recovery after surgery (ERAS) has become a widespread topic in perioperative medicine over the past 20 years. The goals of ERAS are to improve patient outcomes and perioperative experience, reduce length of hospital stay, minimize complications, and reduce cost. Interventions and factors before, during, and after surgery all potentially play a role with the cumulative effect being superior quality of patient care.

Recent Findings Preoperatively, patient and family education, optimization of nutritional status, and antibiotic prophylaxis all improve outcomes. Recovery is also expedited by the use of multimodal analgesia, regional anesthesia, and opioid reducing approaches. Intraoperatively, the anesthesiologist can have an impact by using less-invasive monitors appropriately to guide fluid and hemodynamic management as well as maintaining normothermia. Postoperatively, early enteral feeding, mobilization, and removal of invasive lines support patient recovery. Implementation of ERAS protocol in cardiac surgery faces challenges by some unique perioperative perspectives in cardiac surgery, such as systemic anticoagulation, use of cardiopulmonary bypass, significantly more hemodynamic variations, larger volume replacement, postoperative intubation and mechanical ventilation and associated sedation, and potentially significantly more coexisting morbidities than other surgical procedures.

Summary ERAS in cardiac surgery may benefit patients more related to its high risk and high cost nature. This manuscript specifically reviews the unique aspects of enhanced recovery in cardiac surgery.
\end{abstract}

Keywords Enhanced recovery after surgery $\cdot$ Cardiac surgery · Cardiopulmonary bypass · Pulmonary artery catheterization . Transesophageal echocardiography $\cdot$ Carbohydrate loading $\cdot$ Antifibrinolytic agents $\cdot$ Sternal wound infection

\section{Introduction}

Enhanced recovery after surgery (ERAS) has increasingly become a popular topic in perioperative medicine. The goals of ERAS are to reduce the length of hospital stay, minimize complications, reduce costs, and eventually improve patients'

This article is part of the Topical Collection on Other Pain

Henry Liu

henryliupa@gmail.com

Richard D. Urman

rurman@bwh.harvard.edu

1 Department of Anesthesiology and Perioperative Medicine,

Hahnemann University Hospital, Drexel University College of

Medicine, 245 N. 15th Street, MS 310, Philadelphia, PA 19102, USA perioperative experience and satisfaction. Implementation of ERAS requires a multidisciplinary approach and seamless transition of care from preoperative counseling to intraoperative monitoring and to postoperative management $[1 \bullet \bullet, 2 \bullet \cdot]$. ERAS protocols have been successful with other types of surgeries such as colorectal and orthopedic surgery $[2 \bullet, 3]$. Though the
2 Department of Anesthesiology, Hubei Women and Children's Hospital, 745 Wuluo Road, Hongshan, Wuhan 430070, Hubei, China

3 Department of Anesthesiology, Wuhan Central Hospital, 26 Shengli Street, Jiang'an District, Wuhan 430072, Hubei, China

4 Department of Anesthesiology, Louisiana State University Health Sciences Center, Room 656, 1542 Tulane Ave, New Orleans, LA 79112, USA

5 Department of Anesthesiology, Perioperative and Pain Medicine, Brigham and Women's Hospital, Boston, MA, USA 
concept of "Fast-track surgery" was initially recommended in cardiac surgery, comprehensive ERAS protocol in cardiac surgery has been lagging behind other surgical specialties (gastrointestinal surgery). ERAS protocol in cardiac surgery is recently gaining popularity and appears to improve patient outcomes $[2 \bullet \bullet, 4]$. The implementation of ERAS in cardiac surgery can also have significant financial implications, as cardiovascular patients have the single highest cost center. [5].

\section{Surgical Stress Response}

The majority of elements associated with ERAS programs focus on ways to reduce stress in the perioperative period. Stress and injury cause an inflammatory response and alter the immune system function. Direct cellular injury causes release of pro-inflammatory molecules such as TNF- $\alpha$ and interleukins 1 , 6 , and 8 . The inflammatory cascade is sensed in the central nervous system, and stress response hormones such as corticotrophin are released leading to a catecholamine surge $[2 \bullet \bullet, 6]$. This surge can lead to hemodynamic instability and myocardial injury $[7,8]$. Insulin resistance and increased glucose production are an additional concern as a result of the hormonal changes [6]. This pro-inflammatory process is particularly important for cardiac surgery because of the inflammation-inducing nature of cardiopulmonary bypass (CPB) [9]. In fact, CPB produces an immune response similar to what occurs in sepsis or systemic inflammatory response syndrome [7].

\section{Preoperative Preparations}

\section{Counseling and Education}

Educating the patient and their support system is a key part of the ERAS process. This helps to set expectations, which can reduce anxiety and increase patient compliance. Topics to be discussed include use of incentive spirometer, perioperative nutrition, early mobilization after surgery, smoking cessation, and alcohol use. Abstinence from tobacco and alcohol 1 month prior to surgery improves wound healing [10]. Patient training with incentive spirometry will improve compliance after surgery and reduce incidence of pulmonary complications [11••]. During a preoperative evaluation, patients should also be encouraged to increase their physical activity prior to surgery. This "physical pre-habilitation" helps expedite recovery of functional status after surgery $[10,12]$. Intuitively, patients in better physical condition prior to surgery have better outcomes. Even patients with restrictive co-morbidities such as heart failure, coronary artery disease, and chronic obstructive pulmonary disease can make improvements to their functional status prior to surgery [12].

\section{Nutrition}

Preoperative malnutrition is associated with more postsurgical complications, increased length of stay, higher readmission rates, and slower return of gastrointestinal function. Patients should be screened for malnutrition prior to surgery. Objective assessment of poor nutritional status can be done with screening questionnaires. If malnutrition is suspected, consultation of a dietician is recommended [2••]. Total parenteral nutrition is not recommended unless the patient is severely malnourished [12].

\section{Fasting}

Traditionally, fasting has been a major part of preoperative care to reduce the risk of aspiration during anesthesia. However, fasting contributes to a catabolic state that is already taking place due to the stress response of surgery. Fasting leads to breakdown of proteins and fats and depletes glycogen stores for energy needs $[12,13]$. Therefore, decreasing the amount of time fasted prior to surgery is an important piece to improving patient outcomes and satisfaction.

\section{Preoperative Carbohydrate}

Carbohydrate loading prior to surgery with enteral liquids has been shown to help optimize nutritional status prior to surgery. In fact, carbohydrate intake has been shown to decrease postoperative insulin resistance, surgical site infections, total number of complications, hospital length of stay, and hasten the return of bowel function $[12,13]$. Most protocols recommend a form of glucose-rich drink 2-3 h prior to surgery and some also recommend a carbohydrate drink the night before surgery $[10,14]$. Clearfast, Powerade, and Gatorade are common choices for most ERAS programs. In one study, $400 \mathrm{~mL}$ of carbohydrate-rich drink was cleared from the stomach within $90 \mathrm{~min}$ [13]. Therefore, consuming these drinks $2-3 \mathrm{~h}$ prior to surgery will allow sufficient time for complete gastric emptying. However, careful consideration should be given for patients with diabetic gastroparesis, gastroesophageal reflux disease, or other disorders of gastric motility [13].

\section{Antibiotics}

Surgical site infections can be decreased by intravenous administration of antibiotics $30-60 \mathrm{~min}$ prior to incision and the use of skin antiseptic cleaning solutions [10]. According to the Society of Thoracic Surgeons practice guidelines, a betalactam antibiotic is recommended $30 \mathrm{~min}$ prior to incision. However, allergies, known resistance, or known organisms may dictate a more appropriate drug. Medications such as vancomycin and fluoroquinolones, which are used commonly, should be given within $2 \mathrm{~h}$ of incision [15]. Antibiotics should 
be continued for $48 \mathrm{~h}$ as this has been shown to decrease sternal surgical site infections. However, after $48 \mathrm{~h}$, the benefit is not greater, but the risk of secondary infections such as Clostridium difficile increases [15, 16]. Screening for methicillin-resistant Staphylococcus aureus (MRSA) can also have an impact on outcomes. If patients known to carry MRSA are treated preoperatively with nasal and topical antibiotics, surgical site infections are reduced [17].

\section{Factors Predicting ERAS Failure}

ERAS protocol is not good for all patients. There are some predictors found to be useful to choose patients to be enrolled in ERAS protocol. Youssefi et al. found some factors which can predict failure in fast-track cardiac surgery. These predictors of failure included renal failure, hypertension, age, abnormal EuroSCORE, cardiopulmonary bypass time, first lactate or base deficit after surgery, and cross-clamp time. They unveiled that the strongest predictor of failure was glomerular filtration rate $(\mathrm{GFR})<65 \mathrm{~mL} / \mathrm{min} / \mathrm{BSA}$, with a $54 \%$ sensitivity and $61 \%$ specificity [18].

\section{Intraoperative Management}

\section{Invasive Monitoring}

Pulmonary artery catheters (PAC) are a common invasive monitor used in cardiac surgery. They provide cardiac output, mixed venous oxygen saturation, central venous pressure (CVP), and pulmonary capillary wedge pressure (PCWP) measurements. CVP and PCWP are used as proxies of ventricular function and volume status to aid in the care of critically ill patients. However, cardiac surgery alters myocardial compliance and makes CVP/PCWP less accurate [19]. Furthermore, errors in interpretation and management based on this information are not infrequent [20]. This has led many to question the utility of these monitors. In fact, placement of PACs has led to increased operative mortalities, more endorgan complications, delayed extubation, and increased length of stay in multiple studies [21, 22••]. Additionally, $\mathrm{Xu}$ et al. found that PACs significantly increased cost of admission without a morbidity or mortality benefit [23].

Transesophageal echocardiography (TEE) is one of the most important monitors in cardiac surgery. TEE provides direct visualization of the aorta, cardiac function, volume status, valve structure and function, and the presence of shunts [19, 24]. TEE is less invasive than PAC and provides more reliable information [19, 24]. Mixed venous oxygen saturation is the only piece of information a PAC provides that TEE cannot $[2 \bullet \bullet]$.

\section{Fluid Management}

Management of volume status in cardiac patients is made simpler with invasive monitoring. As mentioned above, CVP and PCWP play an important role, but TEE is the most important monitor for fluid management and choice of vasopressor support [22••, 24]. The goal of intraoperative fluid administration is euvolemia determined by the clinician using the above-mentioned invasive monitors. Hypovolemia leads to hypoperfusion and end organ dysfunction. Hypervolemia disrupts glycocalyx integrity and leads to increased fluid in the interstitium. Volume challenges are recommended when hypovolemia is suspected in order to gauge responsiveness. Balanced crystalloid solutions offer the best profile for patients [25]. The clinician must be aware that patients have a tendency toward volume overload because the CPB circuit requires intravenous fluid for priming [2••].

\section{Blood Products}

Avoidance of blood product transfusions is ideal if possible, because blood products are pro-inflammatory and expose patients to risk of infection and transfusion reactions [7]. Cardiac surgery patients are at a higher risk for need of blood products. Modified ultrafiltration is one strategy to reduce the need for blood transfusions by returning whole blood to the patient after removal of inflammatory mediators created by the CPB circuit [26]. Modified ultrafiltration does decrease inflammatory elements, blood loss, and need for transfusions. However, intensive care unit (ICU) and hospital length of stay did not improve in one study. Modified ultrafiltration is well studied in pediatric patients and shown to improve morbidity, but further studies are needed in adults to find a similar effect if one exists [27]. Intraoperative cell salvage also reduces circulating inflammatory mediators and the need for blood transfusions. This results in lower medical costs, transfusion complications, and postoperative pulmonary complications [28].

\section{Anti-fibrinolytics}

According to the Society of Cardiovascular Anesthesiologists practice guidelines, anti-fibrinolytics are strongly recommended for cardiac surgery [29]. Three such drugs have been studied and clinically used in this regard: aprotinin, aminocaproic acid, and tranexamic acid. Aprotinin is a serine protease inhibitor, which reduces blood loss and transfusion requirements shown by a multicenter double-blind clinical trial [30, 31]. Aminocaproic acid and tranexamic acid alter the ability of plasmin to dissolute thrombin clots. Multiple studies demonstrate the equal capability of these drugs to decrease blood loss and need for transfusions. However, aprotinin has been linked to an increased risk of long-term mortality after coronary artery bypass graft surgery [32]. 
Aprotinin was pulled out of market in 2007 , but recently in Europe, there are studies in attempt to bring this potent antifibrinolytic agent back to the market [29]. Adverse events associated with anti-fibrinolytics are due to thromboembolic sequelae and the risk of seizures [33].

\section{Temperature Management}

Temperature management is different from many other surgical procedures, because intentional hypothermia is often used during $\mathrm{CPB}$ as a neuroprotective and cardioprotective strategy. However, normothermia (>36 C) should be maintained before and after CPB. Hypothermia after weaning off CPB may lead to platelet dysfunction, increased need for transfusions, coagulopathy, and increased bleeding [34]. Hypothermia has multiple additional systemic adverse effects including myocardial depression, arrhythmias, pulmonary hypertension, altered drug metabolism, increased surgical site infections, and increased mortality. All of these complications increase length of stay and healthcare costs $[10,35]$. Use of forced air warming blankets, fluid warmers, and increased room temperature helps to prevent hypothermia.

\section{Hemodynamic Management}

When hemodynamic instability, primarily hypotension and low cardiac output, is encountered in the operating room, there are multiple cardioactive, vasoactive drugs from which to choose. Making the best choice will usually depend upon information gathered from invasive monitors, institutional tradition, and physicians' clinical judgment. See Table 1 for a summary of the effects of specific vasoactive drugs [9, 31, 36]. Maintaining relatively stable hemodynamic parameters ( $\leq 20 \%$ deviation from the baseline) is critical for the success of ERAS protocol and patients' clinical outcomes [37].

\section{Anesthetic Management}

The induction of anesthesia can cause drastic hemodynamic changes. Post-induction hypotension leads to increased morbidity and mortality, and patients with cardiac disease are more susceptible to such sequelae [37]. This is particularly a concern for patient with left ventricular dysfunction or valvular disorders intolerant to afterload reduction (aortic and mitral stenosis). Etomidate is an appropriate choice for patients with poor left ventricular function because of its relatively stable cardiovascular profile compared to propofol. Though etomidate can produce adrenal suppression, this affect does not appear to be clinically relevant after an induction dose of the drug $[37,38]$. Ketamine will maintain afterload, but it is a negative inotrope and may cause severe hypotension in catecholamine-depleted patients [37]. The choice of volatile anesthetic does not appear to affect clinical results. Isoflurane and sevoflurane were found to have no difference in patient outcomes [39].

\section{Blood Glucose Management}

Insulin resistance is a constant concern during and after cardiac surgery even for non-diabetic patients. In fact, hyperglycemia occurs in more than half of patients after coronary artery bypass graft surgery with or without a history of diabetes. Hyperglycemia is linked with multiple complications including infection, bleeding, arrhythmias, and cerebral edema leading to extended length of hospital stay and increased healthcare cost $[40,41]$. Limiting hyperglycemia is important to improve outcomes, but hypoglycemia can also be detrimental. Current evidence regarding glucose control is inconclusive. However, maintaining a blood glucose level of 100 $180 \mathrm{mg} / \mathrm{dL}$ seems a safe approach with a goal of glucose less than $150 \mathrm{mg} / \mathrm{dL}[2 \cdot \bullet, 40]$.

\section{Multimodal Pain Regimen}

Pain control is a key portion of any ERAS protocol. Patient satisfaction and perioperative experience are directly linked with perioperative pain control. Poor analgesia can lead to tachycardia, hypertension, immunosuppression, platelet dysfunction, increased catabolism, and prolonged immobility $[2 \bullet \bullet, 8]$. Pain control should be multimodal and opioid utilization should be minimized when possible $[1 \bullet \bullet, 2 \bullet \cdot$. Perioperative multimodal analgesia can be achieved with pharmacological management (acetaminophen, gabapentin, non-steroidal anti-inflammatory drugs, ketamine, opioids, alpha $a_{2}$-adrenergic drugs, and regional blocks) and nonpharmacological techniques (TENS, relaxation, hypnosis, acupuncture etc.) $[2 \cdot \bullet]$.

The evidence for neuraxial techniques is mixed. Multiple studies have shown that a high thoracic epidural catheter can provide multiple benefits including decreased stress response, increased coronary perfusion leading to less ischemia and arrhythmias, improved pain control, and less pulmonary complications. This is thought to be due to the sympathectomy and analgesia it provides [8, 42]. However, other studies have demonstrated no improvement in morbidity in cardiac patients at 1 month after surgery with a thoracic epidural [43]. This difference may be reconciled by what the epidural is being compared against. Newer protocols for these patients include fast-track extubation and shorter acting sedatives, which might have improved complication rates and likely diminished the benefits of a thoracic epidural [8]. Epidurals are not without complications. The risk of epidural hematoma in cardiac patients is poorly defined but likely with rates ranging between 1 in 1500 and 1 in 150,000 [8].

Regional anesthetic techniques also offer improved pain control. Paravertebral blocks are injection of local anesthetic 
Table 1 Agents affecting cardiovascular activities

\begin{tabular}{llll}
\hline Drug & Contractility & Heart rate & Systemic vascular resistance \\
\hline Epinephrine & $\uparrow \uparrow \uparrow$ & $\uparrow \uparrow \uparrow$ & $\uparrow \uparrow$ \\
Norepinephrine & $\uparrow \uparrow \uparrow$ & Reflexive bradycardia & $\uparrow \uparrow \uparrow$ \\
Dopamine & $\uparrow$ & $\uparrow$ & $\uparrow$ (at higher doses) \\
Dobutamine & $\uparrow$ & $\uparrow \uparrow \uparrow$ & $\uparrow \downarrow$ \\
Phenylephrine & & Reflexive bradycardia & $\uparrow$ \\
Vasopressin & & & $\uparrow \uparrow \uparrow$ \\
Milrinone & $\uparrow \uparrow$ & $\uparrow$ & $\downarrow \downarrow$ \\
Isoproterenol & & $\uparrow \uparrow \uparrow$ & $\downarrow$ \\
\hline
\end{tabular}

$\uparrow$ or $\downarrow=$ mild change, $\uparrow \uparrow$ or $\downarrow \downarrow=$ moderate change, $\uparrow \uparrow \uparrow$ or $\downarrow \downarrow \downarrow=$ marked change agent(s) adjacent to the vertebral canal where the spinal nerve exits. Intercostal nerves can be blocked under direct visualization prior to closing the chest to provide improved analgesia and pulmonary function after surgery. Intrapleural blocks involve local anesthetic between the visceral and parietal pleura via bolus or catheter infusion. Care must be taken to avoid systemic toxicity with this technique. These blocks have fewer complications than neuraxial techniques and coagulopathy is much less of a concern [8].

In theory, NSAIDs can have a potential benefit as both an analgesic and anti-inflammatory medication. Studies have shown NSAIDs expedite early extubation and mobility without increased risk of bleeding or renal injury in the appropriate patient population. However, the FDA has issued a black box warning against the use of NSAIDs after cardiac surgery. This stems from data showing parecoxib and valdecoxib increased complication rates related to thrombotic events after coronary artery bypass surgery such as pulmonary embolism, stroke, and myocardial infarction [44]. Therefore, the decision to use this class of medications should be made with careful consideration of the risks and benefits.

\section{Anti-inflammatory Medications}

A seemingly obvious choice to reduce the inflammatory response from cardiac surgery is corticosteroids, which are powerful anti-inflammatory medications and being used extensively in clinical practice. However, controlled clinical trials have not shown a clinical benefit to their use. An exception to this is patients with reactive airway disease. In addition to being anti-fibrinolytic, aprotinin reduces the activity of neutrophils and may attenuate an inflammatory response [7]. In the perioperative period, statins have shown to be both cardioprotective and antiinflammatory [45]. Both pravastatin and rosuvastatin have been shown to reduce the inflammatory response to cardiopulmonary bypass $[45,46]$. This appears to be due to a decrease in nitric oxide release.

\section{Postoperative Management}

\section{Hemodynamic Management}

Fluid management for these patients is easier in the operating room with transesophageal echocardiography and invasive monitors such as arterial lines and pulmonary artery catheters readily available to show volume status and guide management accordingly. The goals for these patients' hemodynamic management are the same intraoperatively as it is postoperatively: euvolemia, constant body weight, and stable hemodynamic parameters. Patients have difficulty excreting sodium after surgery so dextrose solutions are preferred to high salt crystalloid solutions. Colloids are more costly than colloids, and colloids have not been shown any benefit in perioperative morbidity. Ultimately, the decision to administer intravenous fluids requires comprehensive clinical judgment $[9,25]$.

\section{Postoperative Nausea and Vomiting}

Patient satisfaction is strongly correlated with postoperative nausea and vomiting. The incidence of postoperative nausea and vomiting in cardiac patients is $20-40 \%$ higher than general surgery patients. This delays recovery, creates electrolyte imbalances, puts stress on surgical incisions, and results in higher healthcare costs [47]. Identifying risk factors and providing prophylactic medications based on the number of factors decreases the incidence of postoperative nausea and vomiting [10]. The four primary risk factors are perioperative opioid use, non-smoker, female, and history of motion sickness or postoperative nausea and vomiting. Treatment options include 5-HT3 antagonists, dexamethasone, scopolamine, perphenazine, diphenhydramine, propofol, droperidol, NK-1 antagonists, and acupuncture techniques [47].

\section{Early Mobilization}

Early mobilization after cardiac surgery is crucial to the success of enhanced recovery. Early mobilization can significantly 
reduce the incidence of complications, improve functional capacity, and decrease hospital length of stay [48]. However, many clinicians are reluctant to mobilize postoperative cardiac patients, concerning that their circulatory homeostasis would have been impaired due to myocardial stunning, fluid shift, and autonomic dysfunction. Cassina et al. evaluated the hemodynamic responses to a two-staged mobilization procedure in 53 consecutive patients. They recorded hemodynamics parameters including the central venous oxygen saturation $(\mathrm{ScvO} 2$ including CVP, lactate level, MAP, HR, right atrial pressure, and arterial oxygen saturation $(\mathrm{SpO} 2)$ before, during, and after the mobilization). They found that all patients successfully completed the mobilization procedure; thus, they believe early mobilization after cardiac surgery is a safe procedure as long as it is done under close hemodynamic and clinical monitoring in the ICU setting [49]. Mechanically ventilated patients are at the highest risk of immobilization. However, these patients can also be mobilized to help improve outcomes [50]. Devastating thrombotic events such as stroke and pulmonary embolism are also reduced with early mobilization $[1 \bullet \cdot$.

\section{Early Nutrition/GI Stimulation}

The benefits of early enteral nutrition have been shown to outweigh any potential drawbacks. Enteral nutrition reduces ICU and hospital duration, infection risk, and mortality rates, especially in critically ill patients [25]. Return of bowel function improves with enteral feeding. This is likely because function of the small bowel is reliant upon adequate levels of amino acids such as glutamine. Therefore, withholding enteral feeding deprives the body of nutrients essential for gastrointestinal function and prolongs gastrointestinal recovery [12].

\section{Infection Control}

Overall incidence of postoperative deep sternal wound infection is $1.8 \%$. Prevention of this complication is crucial because 30-day mortality for patients with deep sternal wound infection is nearly $10 \%$. The mortality rate increased to $25 \%$ if reoperation is required for the infection [51]. Risk factors for infection include use of blood products, advanced age, obesity, diabetes, smoking, prolonged operative or CPB time, renal failure, and use of thoracic artery conduits [51]. As stated above, perioperative antibiotics are recommended for $48 \mathrm{~h}$ after cardiac surgery to reduce infection. Barring suspected or existing infection, antibiotics should be stopped after this time [15]. Faster removal of endotracheal tubes, Foley catheters, and invasive lines limits exposure to possible infection.

Any indwelling catheter or device increases the risk of infection, bacteremia, and sepsis. Timely removal of unneeded invasive devices can decrease the risk of infections by up to $50 \%$ [52].
Contemporary management of cardiac patients often involves fast-track extubation protocols. This encourages extubation within 4-6 h after arrival in the ICU for patients meeting certain criteria. These criteria are variable by institution, but meant to identify which patients are stable for extubation. Benefits of fast-track extubation could include decreased ventilator-associated pneumonia, less sedative medication, quicker time to rehabilitation, decreased ICU length of stay, and decreased cost. Multiple protocols have been able to decrease time to extubation and ICU length of stay without increasing re-intubation rates [52-55].

In summary, enhanced recovery in cardiac surgery is in accelerating phase. There are various hurdles in implementation of ERAS protocol in cardiac surgery, due partially to some unique perioperative perspectives in cardiac surgery, such as systemic anticoagulation, use of cardiopulmonary bypass, significantly more hemodynamic variations, larger volume replacement, postoperative intubation and mechanical ventilation and associated sedation, and potentially significantly more co-existing morbidities that other surgical procedures. Implementation of ERAS protocol in cardiac surgery may benefit patients more related to its high risk and high cost nature.

\section{Compliance with Ethical Standards}

Conflict of Interest Scott R. Coleman, Ming Chen, Srikant Patel, Hong Yan, Marcus Zebrower, Julie A. Gayle, and Henry Liu declare no conflict of interest. Alan D. Kaye, MD PhD serves on the Speakers Bureau of Depomed and Merck. Richard D. Urman MD MBA received research funding from Medtronic.

Human and Animal Rights and Informed Consent This article does not contain any studies with human or animal subjects performed by any of the authors.

Publisher's Note Springer Nature remains neutral with regard to jurisdictional claims in published maps and institutional affiliations.

\section{References}

Papers of particular interest, published recently, have been highlighted as:

•• Of major importance

1.• Soffin E, YaDeau J. Enhanced recovery after surgery for primary hip and knee arthroplasty: a review of the evidence. Br J Anaesth. 2016;117(suppl 3):iii 62-i72 excellent review of literature related to enhanced recovery for primary hip and knee arthroplasty.

2.• Yang L, Kaye A, Venakatesh A, Green M, Asgarian C, Luedi M, et al. Enhanced recovery after cardiac surgery: an update on clinical implications. Int Anesthesiol Clin. 2017;55(4):148-62 excellent review of literature related to enhanced recovery for cardiac surgery. 
3. Miller T, Thacker J, White W, Mantyh C, Migaly J, Jin J, et al. Reduced length of hospital stay in colorectal surgery after implementation of an enhanced recovery protocol. Anesth Analg. 2014;118(5):1052-61.

4. Fleming I, Garratt C, Guha R, Desai J, Chaubey S, Wang Y, et al. Aggregation of marginal gains in cardiac surgery: feasibility of a perioperative care bundle for enhanced recovery in cardiac surgical patients. J Cardiothorac Vasc Anesth. 2016;30(3):665-70.

5. Cooke M, Rickard C, Rapchuk I, Shekar K, Marshall A, Comans T, et al. PC6 acupoint stimulation for the prevention of postcardiac surgery nausea and vomiting: a protocol for a two-group, parallel, superiority randomised clinical trial: table 1. BMJ Open. 2014;4(11):e006179.

6. Scott M, Miller T. Pathophysiology of major surgery and the role of enhanced recovery pathways and the anesthesiologist to improve outcomes. Anesthesiol Clin. 2015;33(1):79-91.

7. Levy JH, Tanaka K. Inflammatory response to cardiopulmonary bypass. Ann Thorac Surg. 2017;75:S715-20.

8. Ziyaeifard M, Azarfarin R, Golzari SA. Review of current analgesic techniques in cardiac surgery. Is epidural worth it? J Cardiovas Thorac Res. 2014;6(3):133-40.

9. Liu H, Tariq R, Liu GL, Yu L. Pharmacological cerebral protection in cardiac surgery: an update. J Anesth Perioper Med. 2017;4(1): 23-37. Published on Dec 28, 2016. https://doi.org/10.24015/ JAPM.2017.0004.

10. Hohenberger H, Delahanty K. Patient-centered care - enhanced recovery after surgery and population health management. AORN J. 2015;102(6):578-83.

11.• Holubar S, Hedrick T, Gupta R, Kellum J, Hamilton M, Gan T, et al. American Society for Enhanced Recovery (ASER) and Perioperative Quality Initiative (POQI) joint consensus statement on prevention of postoperative infection within an enhanced recovery pathway for elective colorectal surgery. Perioperative Med. 2017;6(1):4 excellent joint consensus statement on prevention of postoperative infection within an enhanced recovery pathway for elective colorectal surgery.

12. Gupta R, Gan T. Preoperative nutrition and Prehabilitation. Anesthesiol Clin. 2016;34(1):143-53.

13. Nygren J, Thorell A, Ljungqvist O. Preoperative oral carbohydrate nutrition: an update. Current Opinion in Clinical Nutrition and Metabolic Care. 2001;4(4):255-9.

14. Holubar SD, Hedrick T, Gupta R, Kellum J, Hamilton M, Gan TJ, et al. American society for enhanced recovery (ASER) and perioperative quality initiative (POQI) joint consensus statement on prevention of postoperative infection within an enhanced recovery pathway for elective colorectal surgery. Perioper Med (Lond). 2017;6:4. https://doi.org/10.1186/s13741-017-0059-2.

15. Edwards FH, Engelman RM, Houck P, Shahian DM, Bridges CR. The Society of Thoracic Surgeons practice guideline series: antibiotic prophylaxis in cardiac surgery, part II: antibiotic choice. Ann Thorac Surg. 2006;81:397-404.

16. Andersen N. Antibiotic prophylaxis in cardiac surgery: if some is good, how come more is not better? J Thorac Cardiovasc Surg. 2016;151(2):598-9.

17. Jog S, Cunningham R, Cooper S, Wallis M, Marchbank A, VascoKnight $\mathrm{P}$, et al. Impact of preoperative screening for meticillinresistant Staphylococcus aureus by real-time polymerase chain reaction in patients undergoing cardiac surgery. J Hosp Infect. 2008;69(2):124-30.

18. Youssefi P, Timbrell D, Valencia O, Gregory P, Vlachou C, Jahangiri $\mathrm{M}$, et al. Predictors of failure in fast-track cardiac surgery. J Cardiothorac Vasc Anesth. 2015;29(6):1466-71. https://doi.org/ 10.1053/j.jvca.2015.07.002.

19. Sarosiek K, Kang C, Johnson C, Pitcher H, Hirose H, Cavarocchi $\mathrm{N}$. Perioperative use of the Imacor hemodynamic transesophageal echocardiography probe in cardiac surgery patients. ASAIO J. 2014;60(5):553-8.

20. Whitener S, Konoske R, Mark JB. Pulmonary artery catheter. Best Pract Res Clin Anaesthesiol. 2014;28:323-35.

21. Schwann N, Hillel Z, Hoeft A, Barash P, Möhnle P, Miao Y, et al. Lack of effectiveness of the pulmonary artery catheter in cardiac surgery. Anesth Analg. 2011;113(5):994-1002.

22.• Sangkum L, Liu GL, Yu L, Yan H, Kaye AD, Liu H. Noninvasive and minimally invasive cardiac output measurement: an update. J Anesth. 2016;30(3):461-80. https://doi.org/10.1007/s00540-0162154-9 excellent review of noninvasive and minimally invasive cardiac output measurement.

23. Xu F, Wang Q, Zhang $\mathrm{H}$, Chen $\mathrm{S}$, Ao $\mathrm{H}$. Use of pulmonary artery catheter in coronary artery bypass graft. Costs and long-term outcomes. PLoS One. 2015;10(2):e0117610.

24. Muralidhar K. Utility of perioperative transesophageal echocardiography. Ann Card Anaesth. 2016;19(5):2.

25. Miller T, Roche A, Mythen M. Fluid management and goaldirected therapy as an adjunct to enhanced recovery after surgery (ERAS). Can J Anesth. 2014;62(2):158-68.

26. Luciani G, Menon T, Vecchi B, Auriemma S, Mazzucco A. Modified ultrafiltration reduces morbidity after adult cardiac operations. Circulation. 2001;104(suppl 1):I-253-9.

27. Zakkar M, Guida G, Angelini G. Modified ultrafiltration in adult patients undergoing cardiac surgery. Interact Cardiovasc Thorac Surg. 2014;20(3):415-21.

28. Engels G, van Klarenbosch J, Gu Y, van Oeveren W, de Vries A. Intraoperative cell salvage during cardiac surgery is associated with reduced postoperative lung injury. Interact Cardiovasc Thorac Surg. 2015;22(3):298-304.

29. Gerstein N, Brierley J, Windsor J, Panikkath P, Ram H, Gelfenbeyn $\mathrm{K}$, et al. Antifibrinolytic agents in cardiac and noncardiac surgery: a comprehensive overview and update. J Cardiothorac Vasc Anesth. 2017;31:2183-205.

30. Levy JH, Pifarre R, Schaff HV, Horrow JC, Albus R, Spiess B, et al. A multicenter, double-blind, placebo-controlled trial of aprotinin for reducing blood loss and the requirement for donor-blood transfusion in patients undergoing repeat coronary artery bypass grafting. Circulation. 1995;92(8):2236-44.

31. Liu H, Fox CJ, Zhang S, Kaye A. Cardiovascular pharmacology: an update. Anesthesiol Clin. 2010 Dec;28(4):723-38.

32. Mangano DT, Miao Y, Vuylsteke A, Tudor IC, Juneja R, Filipescu D, et al. Mortality associated with aprotinin during 5 years following coronary artery bypass graft surgery. JAMA. 2007;297(5):471-9.

33. Harvey R, Salehi A. Con: Antifibrinolytics should not be used routinely in low-risk cardiac surgery. J Cardiothorac Vasc Anesth. 2016;30(1):248-51.

34. Schmied H, Reiter A, Kurz A, Sessler D, Kozek S. Mild hypothermia increases blood loss and transfusion requirements during total hip arthroplasty. Lancet. 1996;347:289-92.

35. Sarkar S, Barks J. Systemic complications and hypothermia. Semin Fetal Neonatal Med. 2010;15(5):270-5.

36. Manaker S. Use of vasopressors and inotropes [Internet]. Available: https://www.uptodate.com/contents/use-of-vasopressors-andinotropes. Accessed 31 Oct 2018.

37. Budde A, Mets B. Pro: etomidate is the ideal induction agent for a cardiac anesthetic. J Cardiothorac Vasc Anesth. 2013;27(1):180-3.

38. Kaushal R, Vatal A, Pathak R. Effect of etomidate and propofol induction on hemodynamic and endocrine response in patients undergoing coronary artery bypass grafting/mitral valve and aortic valve replacement surgery on cardiopulmonary bypass. Ann Card Anaesth. 2015;18(2):172.

39. Zorrilla-Vaca A, Núñez-Patiño R, Torres V, Salazar-Gomez Y. The impact of volatile anesthetic choice on postoperative outcomes of cardiac surgery: a meta-analysis. Biomed Res Int. 2017;2017:1-12. 
40. Girish G, Agarwal S, Dutta N, Pratap H, Satsangi D, Tempe D. Glycemic control in cardiac surgery: rationale and current evidence. Ann Card Anaesth. 2014;17(3):222.

41. Reyes-Umpierrez D, Davis G, Cardona S, Pasquel F, Peng L, Jacobs S, et al. Inflammation and oxidative stress in cardiac surgery patients treated to intensive vs Conservative Glucose Targets. J Clin Endocrinol Metab. 2016;102:2016-3197.

42. Svircevic V, Passier MM, Nierich AP, van Dijk D, Kalkman CJ, van der Heijden GJ. Epidural analgesia for cardiac surgery. Cochrane Database Syst Rev. 2013;6:CD006715. https://doi.org/10.1002/ 14651858.CD006715.pub2.

43. Svircevic V, Nierich AP, Moons KG, Diephuis JC, Ennema JJ, et al. Thoracic epidural anesthesia for cardiac surgery: a randomized trial. Anesthesiology. 2011;114(2):262-70. https://doi.org/10.1097/ ALN.0b013e318201d2de.

44. Kulik A, Bykov K, Choudhry N, Bateman B. Non-steroidal antiinflammatory drug administration after coronary artery bypass surgery: utilization persists despite the boxed warning. Pharmacoepidemiol Drug Saf. 2015;24(6):647-53.

45. Castaño M, González-Santos JM, López J, García B, Centeno JE, Aparicio B, et al. Effect of preoperative oral pravastatin reload in systemic inflammatory response and myocardial damage after coronary artery bypass grafting. A pilot double-blind placebo-controlled study. J Cardiovasc Surg. 2015;56(4):617-29.

46. Ozguler I, Burma O, Uysal A, Akbulut H. Rosuvastatin lowers systemic inflammatory response in coronary artery bypass graft accompanied by cardiopulmonary bypass surgery: a randomized controlled study. Clin Invest Med. 2015;38(4):154.
47. Gan T, Diemunsch P, Habib A, Kovac A, Kranke P, Meyer T, et al. Consensus guidelines for the Management of Postoperative Nausea and Vomiting. Anesth Analg. 2014;118(1):85-113.

48. Santos P, Ricci N, Suster É, Paisani D, Chiavegato L. Effects of early mobilisation in patients after cardiac surgery: a systematic review. Physiotherapy. 2017;103(1):1-12.

49. Cassina T, Putzu A, Santambrogio L, Villa M, Licker MJ. Hemodynamic challenge to early mobilization after cardiac surgery: a pilot study. Ann Card Anaesth. 2016;19(3):425-32. https://doi.org/10.4103/0971-9784.185524.

50. Hruska P. Early mobilization of mechanically ventilated patients. Crit Care Nurs Clin North Am. 2016;28(4):413-24.

51. Kubota H, Miyata H, Motomura N, Ono M, Takamoto S, Harii K, et al. Deep sternal wound infection after cardiac surgery. J Cardiothorac Surg. 2013;8(1):132.

52. Cove M, Ying C, Taculod J, Oon S, Oh P, Kollengode R, et al. Multidisciplinary Extubation protocol in cardiac surgical patients reduces ventilation time and length of stay in the intensive care unit. Ann Thorac Surg. 2016;102(1):28-34.

53. Fitch Z, Debesa O, Ohkuma R, Duquaine D, Steppan J, Schneider E, et al. A protocol-driven approach to early extubation after heart surgery. J Thorac Cardiovasc Surg. 2014;147(4):1344-50.

54. Gutsche J, Erickson L, Ghadimi K, Augoustides J, Dimartino J, Szeto W, et al. Advancing Extubation time for cardiac surgery patients using lean work design. J Cardiothorac Vasc Anesth. 2014;28(6):1490-6.

55. Sawatzky J, Rivet M, Ariano R, Hiebert B, Arora R. Post-operative nausea and vomiting in the cardiac surgery population: who is at risk? Heart \& Lung: The Journal of Acute and Critical Care. 2014;43(6):550-4. 